\title{
Presurgical language mapping in bilingual children using transcranial magnetic stimulation: illustrative case
}

\author{
Savannah K. Gibbs, BS, ${ }^{1}$ Stephen Fulton, MD, ${ }^{1,2}$ Basanagoud Mudigoudar, MD, ${ }^{1,2}$ Frederick A. Boop, MD, ${ }^{1,3,4}$ and \\ Shalini Narayana, $\mathrm{PhD}^{1,2,5}$
}

\begin{abstract}
${ }^{1}$ Neuroscience Institute, Le Bonheur Children's Hospital, Memphis, Tennessee; Departments of ${ }^{2}$ Pediatrics, ${ }^{3}$ Neurosurgery, and ${ }^{5}$ Anatomy and Neurobiology, University of Tennessee Health Science Center, Memphis, Tennessee; and ${ }^{4}$ Semmes Murphey Neurologic and Spine Institute, Memphis, Tennessee
\end{abstract}

\begin{abstract}
BACKGROUND Presurgical mapping of eloquent cortex in young patients undergoing neurosurgery is critical but presents challenges unique to the pediatric population, including motion artifact, noncompliance, and sedation requirements. Furthermore, as bilingualism in children increases, functional mapping of more than one language is becoming increasingly critical. Transcranial magnetic stimulation (TMS), a noninvasive brain stimulation technique, is well suited to evaluate language areas in children since it does not require the patient to remain still during mapping.

OBSERVATIONS A 13-year-old bilingual male with glioblastoma multiforme involving the left parietal lobe and deep occipital white matter underwent preoperative language mapping using magnetic resonance imaging-guided TMS. Language-specific cortices were successfully identified in both hemispheres. TMS findings aided in discussing with the family the risks of postsurgical deficits of tumor resection; postoperatively, the patient had intact bilingual speech and was referred for chemotherapy and radiation.

LESSONS The authors' findings add to the evolving case for preoperative dual language mapping in bilingual neurosurgical candidates. The authors illustrate the feasibility and utility of TMS as a noninvasive functional mapping tool in this child. TMS is safe, effective, and can be used for preoperative mapping of language cortex in bilingual children to aid in surgical planning and discussion with families.
\end{abstract}

https://thejns.org/doi/abs/10.3171/CASE21391

KEYWORDS transcranial magnetic stimulation; language mapping; brain tumor; pediatric; bilingual; noninvasive

Presurgical mapping of eloquent cortex is especially critical in pediatric patients with brain tumors in whom in addition to being an effective treatment, early surgical intervention has the potential to minimize cognitive and neuropsychiatric sequelae and improve overall quality of life. ${ }^{1-4}$ Given that nearly $22 \%$ of American children speak a language other than English at home, ${ }^{5}$ with two-thirds of the world's population speaking two or more languages, ${ }^{6}$ an increasing number of neurosurgical candidates present with language-specific concerns best addressed by noninvasive presurgical mapping. While motor and somatosensory mapping can be performed in children using functional magnetic resonance imaging (fMRI) and magnetoencephalography (MEG), language mapping in this population can be challenging. ${ }^{7-9}$ MEG and $\mathrm{fMRI}$ in children are often performed under sedation, limiting differential languagespecific mapping and often precluding localization of language functions altogether. ${ }^{10}$ Direct cortical stimulation (DCS) has limited success in younger children and is rarely performed in patients with malignant tumor due to time sensitivity and increased risk of complications. $^{11-13}$ On the other hand, transcranial magnetic stimulation (TMS), a noninvasive alternative to DCS, is well suited to evaluate language areas in children, since it is unaffected by patient movement, can be performed with young children, and can be repeated to test multiple languages as necessary. ${ }^{14-17}$ Here, we report a case managed at an academic teaching hospital where TMS was used to localize bilingual language cortices in a 13 -year-old child with glioblastoma multiforme.

ABBREVIATIONS DCS = direct cortical stimulation; EEG = electroencephalogram; $\mathrm{fMRI}$ = functional magnetic resonance imaging; $\mathrm{MEG}$ = magnetoencephalography; $\mathrm{MRI}=$ magnetic resonance imaging; PET = positron emission tomography; TMS = transcranial magnetic stimulation.

INCLUDE WHEN CITING Published October 4, 2021; DOI: 10.3171/CASE21391.

SUBMITTED July 2, 2021. ACCEPTED July 26, 2021.

(C) 2021 The authors, CC BY-NC-ND 4.0 (http://creativecommons.org/licenses/by-nc-nd/4.0/). 


\section{Illustrative Case}

A 13-year-old right-handed English-Italian bilingual White male patient, previously in good health, presented with complaints of generalized tonic-clonic seizure of 20 - to 30 -minute duration to a community hospital. Based on clinical examination and magnetic resonance imaging (MRI), he was initially diagnosed with a left parietal tumor and started on antiseizure medication, levetiracetam. However, seizures continued after discharge with 3-day electroencephalogram (EEG) monitoring capturing up to five electrographic seizures per hour, characterized primarily by word-finding and articulation difficulties, sensations of "fuzziness" in the left posterior head region, and progressive right visual field defects. The patient was referred to our facility for further workup and possible surgical intervention, at which time symptoms had persisted for 6 weeks and had not responded to treatment with levetiracetam or oxcarbazepine. Upon clinical examination, the patient was awake, alert, oriented, and in no distress. Motor and sensory examination was normal, although right inferior quadrantanopia was noted.

\section{Diagnostic Imaging}

MRI of the brain showed a large T2 hyperintense-enhancing mass in the left superior parietal lobule and cuneus, extending anteriorly into the posterior left angular gyrus and medially into the deep white matter of the right occipital lobe (Fig. 1). In addition to confirming the initial diagnosis, the MRI indicated overall growth of 9 $\mathrm{mm}$ as well as slight intratumoral hemorrhage since the initial MRI 6 weeks earlier. Video EEG monitoring showed nearly continuous left temporoparietal focal slowing, focal epileptiform discharges over the left parietal and temporal regions, intermittent bursts of quasirhythmic theta activity over the left parietal region without any associated clinical symptoms, and a single electroclinical seizure arising from the left parietotemporal region.

In addition, the patient underwent fMRI, TMS, and formal visual field testing for localizing motor, language, and vision functions. Using an active-motion paradigm, fMRI localized the upper-extremity motor cortex to the precentral gyrus in both hemispheres, well removed from the lesion; TMS motor mapping results were in agreement. Using monolingual (English) active and passive paradigms, fMRI then localized left expressive language to the anterior and medial tumor margins, with receptive language nearby; however, activation was found in both hemispheres during each task,
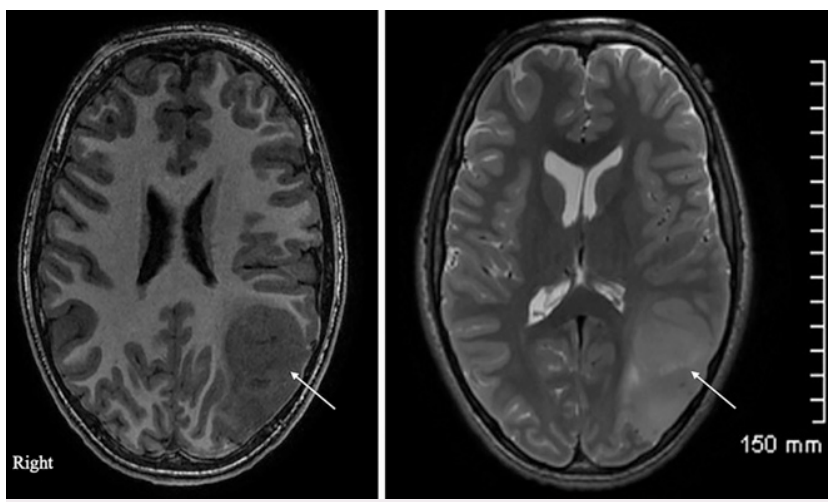

FIG. 1. Preoperative MRI showing T1 (left) and hyperintense T2 (right) mass (white arrows), subsequently identified as glioblastoma multiforme. The images are depicted in radiological convention. suggesting bilateral language representation. Because Italian-language stimuli for fMRI tasks were not readily available, second-language mapping was not performed using $\mathrm{fMRI}$ in this patient. fMRI also indicated early involvement of the tumor in left hemispheric visual field pathways, producing right inferior quadrantanopia, which was confirmed by formal visual field testing.

TMS was used to localize language-specific cortex by employing the "virtual" lesion paradigm. ${ }^{16,18}$ TMS language mapping was performed using the speech module incorporated in the navigated TMS system (NexSpeech module; Nexstim Inc.) and a cooled figure-eight TMS coil. The patient's head was coregistered to the high-resolution T1-weighted MRI without contrast using anatomical landmarks and the surface matching procedure implemented in the TMS system. We first used a standard object naming task commonly used with adolescents and adults, asking the patient to name images in English, and later repeated a simpler version of the test with colors and numbers, asking the patient to name images in Italian. Language mapping was completed in two sessions, one per language, of approximately 60 minutes each; sessions were conducted on day 1 and day 5 of admission, respectively. The Italian-language testing was simplified for ease of analysis, and all images and associated correct responses were confirmed by the patient's mother, a native Italian speaker. Five-hertz TMS (5 pulses) at an intensity of $35 \%$ stimulator output (Electric-field of $75 \mathrm{~V} / \mathrm{m}$ ), time-locked to stimulus onset, was delivered to the temporal lobes in the two hemispheres as the patient performed each task. For each trial, the stimulus presented, the patient's response, and the cortical location of TMS were recorded and compared with his baseline speech performance. When tested in both English and Italian, in the left hemisphere, TMS-induced speech arrest, semantic errors, and hesitation errors were noted in the angular gyrus and the posterior parts of the superior and middle temporal gyri, partially localizing along the anterior and ventral margins of the tumor; additional errors were noted in the anterior superior temporal sulcus during Italian-language testing only (Fig. 2A and B). Stimulation of temporal regions in the right hemisphere revealed speech arrest and hesitation errors in the supramarginal gyrus, posterior superior temporal gyrus, and anterior middle temporal gyrus (Fig. $2 \mathrm{C}$ and D), indicating the presence of English and Italian language-specific cortex in both hemispheres (Fig. 3). Based on these findings, the medical team discussed the risks and benefits of surgery and the possibility of retaining language function with the family and recommended to pursue a microsurgical tumor resection followed by chemotherapy and radiation.

\section{Surgical Procedure}

One week after admission, the patient underwent a stealth frameless stereotactic MRI-guided left parietal craniotomy under general anesthesia. First, microsurgical tumor resection was performed with subcortical white matter tract debulking. Next, intraoperative electrocorticography was performed using four-contact subdural electrodes along the resection cavity $(0.5$ minimum alveolar concentration isoflurane and 0.3 minimum alveolar concentration sufentanil), finding low amplitude mixed delta, theta, and faster frequency activities without epileptiform discharges. An intraoperative MRI was then performed, which showed infiltrated tissue remaining superior to the resected area. Further debulking was performed, after which a second intraoperative MRI indicated total resection of visible tumor but a remainder of infiltrated brain, left in place due to speech involvement (Figs. 2A, 2B, 3A, and 4). Postoperative MRI showed expected surgical changes (Fig. 4). Pathology found poorly differentiated glial cells 


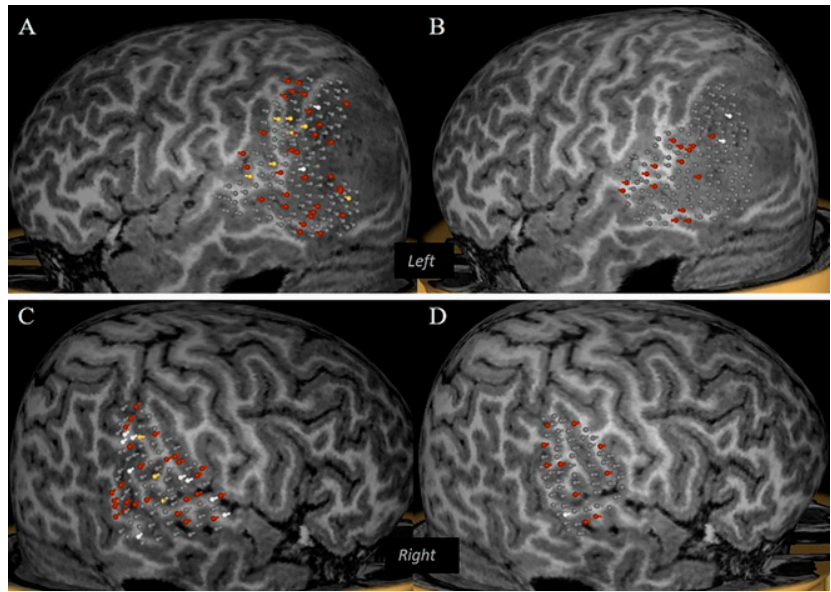

FIG. 2. Results of TMS of the left hemisphere in English (A) and Italian (B) and the right hemisphere in English (C) and Italian (D). Gray pegs indicate stimuli that did not cause a speech error, whereas red pegs indicate performance errors, yellow pegs indicate semantic errors, and white pegs indicate speech arrest.
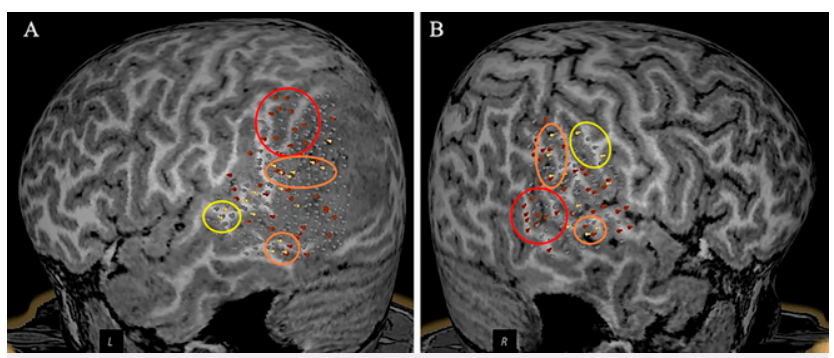

FIG. 3. English and Italian speech maps visualized together in the left (A) and right (B) hemispheres. Red pegs indicate errors in English, while yellow pegs indicate errors in Italian. Gray pegs indicate stimuli that did not cause any speech error. Although each language is represented within the same broad cortical regions in both hemispheres, some focal sites appear to be language-specific. English-specific sites are circled in red; TMS stimulation at these sites produced errors in English but not in Italian. Italian-specific sites are circled in yellow; TMS stimulation at these sites produced errors in Italian but not in English. Shared cortical areas, where TMS stimulation produced errors in both languages, are circled in orange.

with microscopic foci of necrosis and TP53 mutation, consistent with glioblastoma multiforme. The patient's postoperative course was uncomplicated, and his speech in both English and Italian remained intact. He was maintained on levetiracetam and lacosamide and referred to an area hospital for follow-up and chemotherapy and radiation treatment.

\section{Discussion}

Although more than half of people worldwide speak two or more languages, cortical representation of language in bilingual and multilingual speakers remains a topic of debate, with specific attention to whether various languages are represented in distinct or universal areas. Neuroimaging studies using fMRI, MEG, and PET have produced mixed results, likely due in part to differences in language proficiency and age of second language acquisition. ${ }^{19-21}$ However, recent neuroimaging findings ${ }^{22,23}$ have included differences in spatial distribution of languages, ${ }^{24}$ differences in language network white matter connections between bilingual and monolingual speakers, ${ }^{25}$ and increased gray matter density in trilingual speakers compared to bilingual speakers. ${ }^{26}$ Likewise, direct cortical stimulation and Wada studies in bilingual and multilingual speakers have shown both grossly common and distinct language-specific cortical and even subcortical areas. ${ }^{19}$ To our knowledge, three previous studies of bilingual language organization have used TMS, all in healthy adults; all demonstrated both shared and distinct languagespecific cortical areas, although one examined the right hemisphere and differences in error rates between the first and second language did not reach statistical significance. ${ }^{17,27,28}$ Additionally, there are two reports of TMS causing or altering involuntary language switching in three patients following application to the left dorsolateral prefrontal cortex. ${ }^{29,30}$ Our TMS results in this patient provide further evidence that bilingual language representation comprises both shared and distinct, language-specific cortical areas.

Despite a number of reports of selective or differential aphasia in bilingual patients following seizure, stroke, or neurosurgery, ${ }^{19,31-36}$ preoperative language mapping of both or all languages spoken by a neurosurgical candidate is relatively uncommon, especially in a pediatric setting. Given the increasing evidence for partially distinct cortical representation of each language, it may no longer be adequate to use the functional map of a bilingual or multilingual patient's first language as a surrogate for their other tongues, particularly with lesions abridging expected language cortex. . $^{32,37}$ However, investigation of multiple languages may not always be feasible. In children, the general challenges of performing language mapping are amplified by the increased time and compliance needed to repeat mapping in a second language. ${ }^{7-10}$ Because it does not require patients to remain still for long periods of time, TMS ameliorates related compliance and sedation concerns, and sessions can be separated to further reduce fatigue and inattention. ${ }^{12,14,15,18}$ Further, TMS stimulation provides causal, not correlative, information, identifying the sites necessary to the language task rather than merely participatory. ${ }^{17,38}$

\section{Observations}

This case represents the challenges of managing a bilingual child with malignant brain tumor.

While both fMRI and TMS were successful in mapping English-language cortex, second-language mapping in this child was performed with only TMS. Critical bilingual language cortices were successfully identified in both temporal lobes using TMS, and distinct language-specific sites were visualized within common language areas. In this child, bilateral language representation most likely indicated partial diseaseinduced reorganization, and this finding aided in discussion of the risks of surgical intervention, as well as the likelihood of preserved language postoperatively. TMS findings also helped to inform surgical planning, such that function in each of the patient's languages, and consequently the patient's quality of life, could be maximally preserved. Consistent with our expectations, the patient did not have functional deficits in either language following surgery. To the best of our knowledge, this is both the first demonstration of distinct, language-specific cortex in a bilingual clinical patient and the first patient with glioblastoma multiforme to undergo successful bihemispheric presurgical language mapping in multiple languages with TMS. 


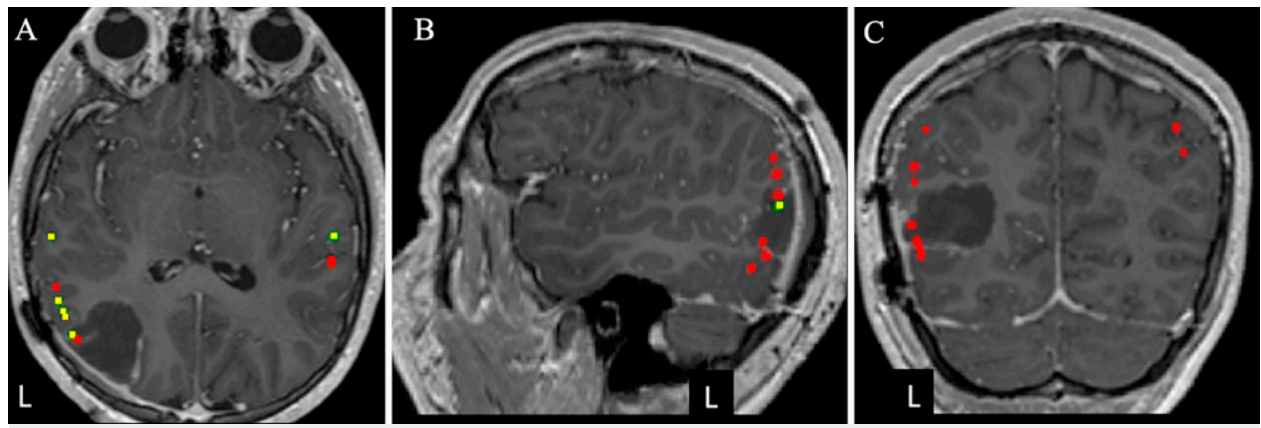

FIG. 4. Postoperative MRI in neurological convention in axial (A), sagittal (B), and coronal (C) views showing locations of TMS responses in English (red) and Italian (yellow) in relation to the resection cavity. The patient had no postoperative deficits in either language.

\section{Lessons}

Neuroimaging and direct cortical stimulation studies of bilingual and multilingual speakers suggest that within common language cortical areas exist more focal, distinct single-language sites. Along with numerous reports of differential or selective aphasia in bilingual speakers following seizure, neurosurgery, or brain lesion, these data imply a need for more detailed examinations of multilanguage representation in bilingual and multilingual patients undergoing neurosurgery. In our patient, TMS was used to map language cortices in English and Italian prior to tumor resection, with language-specific findings within the expected, shared cortical regions. TMS-induced error rates, types, and spatial distributions were different between the two languages, consistent with previous findings. Both language maps were used in consultation with the patient and family, as well as in surgical planning; the patient exhibited no postoperative deficits in either language immediately following surgery or thereafter.

TMS is a safe and effective noninvasive language mapping tool in children and can be readily adjusted for use in bilingual speakers. The primary limitation of the technique for dual language mapping is that investigators must be able to analyze the patient's speech. Ideally, data would be collected and analyzed by investigators fluent in both languages, and the same object naming task and stimuli could be used for both studies; unfortunately, this is not always feasible in a clinical setting. In our case, we instead simplified the stimuli used in the Italian study to include only basic colors and numbers (1-10), so that we could easily learn the correct responses, provided by the patient's mother in order to ensure accuracy and correct for dialectal characteristics, for analysis. Although we have had success using color-naming tasks for clinical language mapping in young and developmentally delayed children, ${ }^{39}$ it is possible that the more rote number-naming task utilized a different network entirely, as recent direct cortical stimulation findings suggest. ${ }^{40}$ Indeed, our patient made no errors when presented with number stimuli during stimulation, and thus we do not recommend use of number-naming tasks for TMS language mapping.

Future large-scale studies are needed to optimize and validate stimuli for use in multiple language mapping with TMS, with particular attention to options for speech analysis by nonfluent investigators.

Another limitation of TMS language mapping, particularly when used to map multiple languages in a single patient, is its time-intensive nature. In our experience, clinical language mapping with TMS takes approximately an hour for the average patient. Each language will require its own TMS session for thorough and comparable mapping, potentially complicating testing with patient fatigue and time or scheduling constraints; we recommend spreading the sessions across several days to mitigate these challenges. Other limitations include TMSinduced muscle activation, primarily with stimulation of the inferior frontal gyri, which may produce jaw or eye twitching, discomfort, and/ or pain, occasionally precluding frontal mapping altogether. Accordingly, lesion location should be taken into account, and patients with lesions in Broca's area or its homolog may benefit from additional language mapping using other methods. Finally, the positive predictive value of TMS compared to direct cortical stimulation varies significantly across studies $(20-97 \%),{ }^{12,14,43,44}$ likely due to lack of standardized methodology. ${ }^{41}$ However, its negative predictive value is consistently very high (74-99\%); thus, TMS has excellent reliability in identifying noneloquent cortex for language. ${ }^{12,14,41,42}$ TMS is also more sensitive, although less specific, than fMRI compared to DCS, and results of TMS are less affected by brain lesions. ${ }^{41,43}$

Our case adds to the growing body of evidence for specialized language areas in bilingual and multilingual speakers, suggesting a potential need for language-specific preoperative mapping in these patients to reduce risks of postoperative selective or differential language impairment. Language-specific mapping during presurgical workup can also aid in the discussion of risks and benefits with patients and families, help to alleviate concerns regarding bilingual function, and assist in accurate, informed surgical planning.

Because TMS is not precluded by patient movement, does not require sedation, is easily adjusted to the patient's own baseline speech patterns, and provides causal, rather than correlative, information, it is a valuable alternative to other noninvasive functional mapping methods, such as MEG and fMRI, especially in children. This report illustrates the feasibility and utility of presurgical TMS language mapping in bilingual children with brain tumor.

\section{Acknowledgments}

We thank Dr. Asim F. Choudhri, neuroradiologist at Le Bonheur Children's Hospital \& University of Tennessee Health Science Center, for his generous contribution of clinical MRI images to this report.

\section{References}

1. Ottenhausen M, Krieg SM, Meyer B, Ringel F. Functional preoperative and intraoperative mapping and monitoring: increasing safety and efficacy in glioma surgery. Neurosurg Focus. 2015;38(1):E3. 
2. Sollmann N, Ille S, Hauck T, et al. The impact of preoperative language mapping by repetitive navigated transcranial magnetic stimulation on the clinical course of brain tumor patients. BMC Cancer. 2015;15(1):261.

3. Krex D, Klink B, Hartmann $C$, et al. Long-term survival with glioblastoma multiforme. Brain. 2007;130(Pt 10):2596-2606.

4. Davis ME. Glioblastoma: Overview of disease and treatment. Clin J Oncol Nurs. 2016;20(5 Suppl):S2-S8.

5. United States Census Bureau. 2017 American Community Survey 1-Year Estimates: Table S1601. Accessed June 1, 2021. https:// data.census.gov/cedsci/table?q=S1601\&tid=ACSST1Y2017.S1601

6. Ramirez NF, Kuhl PK. Bilingual Language Learning in Children. Institute for Learning \& Brain Sciences; 2016.

7. Choudhri AF, Patel RM, Siddiqui A, Whitehead MT, Wheless JW. Cortical activation through passive-motion functional MRI. AJNR Am J Neuroradiol. 2015;36(9):1675-1681.

8. Bercovici E, Pang EW, Sharma R, et al. Somatosensory-evoked fields on magnetoencephalography for epilepsy infants younger than 4 years with total intravenous anesthesia. Clin Neurophysiol. 2008:119(6):1328-1334.

9. Lauronen L, Nevalainen P, Pihko E. Magnetoencephalography in neonatology. Neurophysiol Clin. 2012;42(1-2):27-34.

10. Birg L, Narayana S, Rezaie R, Papanicolaou A. Technical tips: MEG and EEG with sedation. Neurodiagn J. 2013;53(3):229-240.

11. Schevon CA, Carlson $C$, Zaroff $C M$, et al. Pediatric language mapping: sensitivity of neurostimulation and Wada testing in epilepsy surgery. Epilepsia. 2007;48(3):539-545.

12. Lehtinen $\mathrm{H}$, Mäkelä JP, Mäkelä T, et al. Language mapping with navigated transcranial magnetic stimulation in pediatric and adult patients undergoing epilepsy surgery: comparison with extraoperative direct cortical stimulation. Epilepsia Open. 2018;3(2):224-235.

13. Sweet JA, Hdeib AM, Sloan A, Miller JP. Depths and grids in brain tumors: implantation strategies, techniques, and complications. Epilepsia. 2013;54(Suppl 9):66-71.

14. Picht T, Krieg SM, Sollmann N, et al. A comparison of language mapping by preoperative navigated transcranial magnetic stimulation and direct cortical stimulation during awake surgery. Neurosurgery. 2013;72(5):808-819.

15. Narayana S, Papanicolaou AC, McGregor A, Boop FA, Wheless JW. Clinical Applications of Transcranial Magnetic Stimulation in Pediatric Neurology. J Child Neurol. 2015;30(9):1111-1124.

16. Rosenstock T, Picht T, Schneider H, Koch A, Thomale U-W. Left perisylvian tumor surgery aided by TMS language mapping in a 6year-old boy: case report. Childs Nerv Syst. 2019;35(1):175-181.

17. Hämäläinen S, Mäkelä N, Sairanen V, Lehtonen M, Kujala T, Leminen $A$. TMS uncovers details about sub-regional language-specific processing networks in early bilinguals. Neuroimage. 2018;171:209-221.

18. Pascual-Leone A, Walsh V, Rothwell J. Transcranial magnetic stimulation in cognitive neuroscience-virtual lesion, chronometry, and functional connectivity. Curr Opin Neurobiol. 2000;10(2):232-237.

19. Giussani C, Roux FE, Lubrano V, Gaini SM, Bello L. Review of language organisation in bilingual patients: what can we learn from direct brain mapping? Acta Neurochir (Wien). 2007;149(11):1109-1116.

20. Liu H, Cao F. L1 and L2 processing in the bilingual brain: a metaanalysis of neuroimaging studies. Brain Lang. 2016;159:60-73.

21. Kim KHS, Relkin NR, Lee K-M, Hirsch J. Distinct cortical areas associated with native and second languages. Nature. 1997;388(6638):171-174.

22. Kuhl PK, Stevenson J, Corrigan NM, van den Bosch JJF, Can DD, Richards T. Neuroimaging of the bilingual brain: structural brain correlates of listening and speaking in a second language. Brain Lang. 2016;162:1-9.
23. Köpke B, Howells RKR, Cortelazzo F, Péran P, de Boissezon X, Lubrano $V$. Functional and structural differences in brain networks involved in language processing and control in highly proficient early and late bilinguals. J Neurolinguist. 2021;59:100988.

24. Brignoni-Perez E, Jamal NI, Eden GF. An fMRI study of English and Spanish word reading in bilingual adults. Brain Lang. 2020;202:104725.

25. García-Pentón L, Pérez Fernández A, Iturria-Medina Y, GillonDowens M, Carreiras M. Anatomical connectivity changes in the bilingual brain. Neuroimage. 2014;84:495-504.

26. Grogan A, Parker Jones O, Ali N, et al. Structural correlates for lexical efficiency and number of languages in non-native speakers of English. Neuropsychologia. 2012;50(7):1347-1352.

27. Tussis L, Sollmann N, Boeckh-Behrens T, Meyer B, Krieg SM. Identifying cortical first and second language sites via navigated transcranial magnetic stimulation of the left hemisphere in bilinguals. Brain Lang. 2017;168:106-116.

28. Tussis L, Sollmann N, Boeckh-Behrens T, Meyer B, Krieg SM. The cortical distribution of first and second language in the right hemisphere of bilinguals - an exploratory study by repetitive navigated transcranial magnetic stimulation. Brain Imaging Behav. 2020;14(4):1034-1049.

29. Nardone R, De Blasi P, Bergmann J, et al. Theta burst stimulation of dorsolateral prefrontal cortex modulates pathological language switching: a case report. Neurosci Lett. 2011;487(3):378-382.

30. Holtzheimer P, Fawaz W, Wilson C, Avery D. Repetitive transcranial magnetic stimulation may induce language switching in bilingual patients. Brain Lang. 2005;94(3):274-277.

31. Aladdin Y, Snyder TJ, Ahmed SN. Pearls \& Oy-sters: selective postictal aphasia: cerebral language organization in bilingual patients. Neurology. 2008;71(7):e14-e17.

32. Fabbro F. The bilingual brain: bilingual aphasia. Brain Lang. 2001;79(2):201-210.

33. Gomez-Tortosa E, Martin EM, Gaviria M, Charbel F, Ausman JI. Selective deficit of one language in a bilingual patient following surgery in the left perisylvian area. Brain Lang. 1995;48(3):320-325.

34. Ku A, Lachmann EA, Nagler W. Selective language aphasia from herpes simplex encephalitis. Pediatr Neurol. 1996;15(2):169-171.

35. Leemann B, Laganaro M, Schwitter V, Schnider A. Paradoxical switching to a barely-mastered second language by an aphasic patient. Neurocase. 2007;13(3):209-213.

36. Hervais-Adelman AG, Moser-Mercer B, Golestani N. Executive control of language in the bilingual brain: integrating the evidence from neuroimaging to neuropsychology. Front Psychol. 2011;2:234.

37. Lucas TH 2nd, McKhann GM 2nd, Ojemann GA. Functional separation of languages in the bilingual brain: a comparison of electrical stimulation language mapping in 25 bilingual patients and 117 monolingual control patients. J Neurosurg. 2004;101(3):449-457.

38. Weber MJ, Thompson-Schill SL. Functional neuroimaging can support causal claims about brain function. J Cogn Neurosci. 2010;22(11):2415-2416.

39. Narayana S, Gibbs SK, Fulton SP, et al. Clinical utility of transcranial magnetic stimulation (TMS) in the presurgical evaluation of motor, speech, and language functions in young children with refractory epilepsy or brain tumor: preliminary evidence. Front Neurol. 2021;12:650830.

40. Rojas PH, Sivaraju A, Quraishi IH, et al. Electrical cortical stimulation can impair production of the alphabet without impairing counting. Epilepsy Behav Rep. 2021;15:100433.

41. Jeltema H-R, Ohlerth A-K, de Wit A, et al. Comparing navigated transcranial magnetic stimulation mapping and "gold standard" direct cortical stimulation mapping in neurosurgery: a systematic review. Neurosurg Rev. 2021;44(4):1903-1920.

42. Bährend I, Muench MR, Schneider $\mathrm{H}$, et al. Incidence and linguistic quality of speech errors: a comparison of preoperative transcranial 
magnetic stimulation and intraoperative direct cortex stimulation. J Neurosurg. 2020;134(5):1409-1418.

43. Ille S, Sollmann N, Hauck T, et al. Impairment of preoperative language mapping by lesion location: a functional magnetic resonance imaging, navigated transcranial magnetic stimulation, and direct cortical stimulation study. J Neurosurg. 2015;123(2):314-324.

44. Babajani-Feremi A, Narayana S, Rezaie R, et al. Language mapping using high gamma electrocorticography, fMRI, and TMS versus electrocortical stimulation. Clin Neurophysiol. 2016;127(3):1822-1836.

\section{Disclosures}

The authors report no conflict of interest concerning the materials or methods used in this study or the findings specified in this paper.

\section{Author Contributions}

Conception and design: Gibbs, Narayana. Acquisition of data: Gibbs, Narayana. Analysis and interpretation of data: Gibbs, Narayana.
Clinical care and assimilation of imaging and TMS data: Fulton, Mudigoudar, Boop. Drafting the article: Gibbs. Critically revising the article: All authors. Reviewed submitted version of manuscript: All authors. Approved the final version of the manuscript on behalf of all authors: Narayana. Study supervision: Narayana.

\section{Supplemental Information}

Previous Presentations

Portions of this work were presented at the 73rd Annual Meeting of the American Epilepsy Society, Baltimore, MD, December 8, 2019.

\section{Correspondence}

Shalini Narayana: Le Bonheur Children's Hospital, Memphis, TN. snaraya2@uthsc.edu. 